\title{
Combination of berberine and evodiamine inhibits intestinal cholesterol absorption in high fat diet induced hyperlipidemic rats
}

\author{
Xin Zhou ${ }^{1}$, Fengying Ren ${ }^{2}$, Hong Wei ${ }^{1}$, Liyun Liu ${ }^{3}$, Tao Shen ${ }^{* *}$, Shijun $\mathrm{Xu}^{4}$, Jiangping Wei ${ }^{4}$, Jiayue Ren ${ }^{4}$ \\ and Hengfan $\mathrm{Ni}^{4}$
}

\begin{abstract}
Background: Hyperlipidemia characterized of elevated serum lipid levels is a prevalent disease frequently resulting in cardiovascular disease (CVD). Berberine and evodiamine are herbal products of traditional Chinese herb Coptis chinensis and Evodia rutaecarpa, which are indicated to exert regulation of lipid metabolism. Therefore, the objective of this study was to investigate the lipid-lowering effect of berberine and evodiamine combination in hyperlipidemic rats.
\end{abstract}

Method: The rat model of hyperlipidemia was established by providing high-fat-diet (HFD) for 4 weeks. Berberine (BB), evodiamine (EV), and their combination (BB + EV) were orally administered to HFD induced rats for 4 weeks. Body weight, food utilization, histopathology of liver tissues, lipid profiles of serum and liver were measured. Gas chromatography (GC) analysis was applied to examine the level of plasma total cholesterol and B- Sitosterol (BS) to estimate cholesterol absorption activity. Furthermore, intestinal NPC1L1, ACAT2, and ApoB48 protein expressions were evaluated by immunohistochemical assay.

Result: According to the results, decreased levels of serum cholesterol (TC), triglycerides (TG), low density lipoproteincholesterol (LDL-C), as well as hepatic TC were showed in hyperlipidemic rats treated by combination of berberine and evodiamine. GC analysis indicated that the elevated plasma BS was significantly ameliorated by BB, EV, and BB + EV. In addition, immunohistochemical analysis revealed that $\mathrm{BB}+\mathrm{EV}$ treatment down-regulated the expressions of intestinal NPC1L1 and ACAT2, and ApoB48 in HFD induced rats.

Conclusion: Based on the above results, combination of berberine and evodiamine exerted a promising preventive effect on hyperlipidemia, partially through inhibiting intestinal absorption of cholesterol.

Keywords: Berberine, Evodiamine, Hyperlipidemia, Cholesterol absorption, NPC1L1, ACAT2, ApoB48

\section{Background}

Hyperlipidemia is an abnormality of lipid metabolism, characterized by an elevation of total cholesterol (TC), triglyceride (TG), and low density lipoprotein-cholesterol (LDL-C), and/or a decreasing of high density lipoproteincholesterol (HDL-C) in circulating levels [1]. Cholesterol homeostasis plays a notable role in maintaining blood lipid level. A large number of studies have demonstrated that disruption of cholesterol metabolism is a high risk of

\footnotetext{
* Correspondence: shentaotcm@aliyun.com

${ }^{1}$ School of Basic Medicine, Chengdu University of Traditional Chinese Medicine, Chengdu 611137, China

Full list of author information is available at the end of the article
}

cardiovascular disease (CVD) [2, 3]. Absorption of cholesterol in intestine is a significant physiological process which counts for the transportation of chylomicron cholesterol to liver, involved in maintaining lipid homeostasis [4]. In view of the close relationship between cholesterol absorption and lipid metabolism, cholesterol absorption pathway has been recognized as an important pharmacological intervention for the therapeutic target of hyperlipidemia [5]. Thus, the prevention of hyperlipidemia can be achieved by reducing the intestinal absorption of cholesterol [6].

Berberine is an alkaloid found in the roots of Coptidis Rhizoma which is a traditional Chinese medicine known 
as Huanglian. Berberine has been testified to exert various biological effects including anti-inflammatory, anticancer,anti-microbial, and anti-diabetic activities [7-10]. Extensive numbers of studies have confirmed berberine with its hypoglycemic and hypolipidemic effect in clinical and experimental researches $[10,11]$. It has been reported that administration with berberine by gavaging for 8 weeks could reduce serum total cholesterol level by 29\%-33\% through inhibiting the intestinal absorption in high fat diet induced rats [12]. Evodiamine, the major bioactive component found in Evodia rutaecarpa, possess beneficial pharmacological effects involving anticancer, anti-inflammation, anti-obesity and anti-diabetes effects [13-17]. Previous research has reported that evodiamine could influence lipid metabolism through regulation of the expressions of peroxisome proliferatoractivated receptor-g (PPARg) in mouse adipose and liver tissues [18]. Furthermore, Chao DC et al. found that coadministration of berberine and evodiamine could suppress tumor promotion primarily through AP-1 and/or NF- $\mathrm{B}$ p pathways in HepG2 cells [19]. Previous research of our laboratory has found that berberine concomitant with evodiamine had ameliorative effect in high fat diet induced rats. However, the effect of the combination of berberine and evodiamine on modulation of cholesterol absorption has not been experimentally researched. As for the lipid-regulated effects of the two drugs, exploration of the combination of berberine and evodiamine on modulation of lipid metabolism through cholesterol absorption would provide interesting results.

Therefore, the present study was undertaken to determine whether the combination of berberine and evodiamine exerted their protective effect on hyperlipidemia induced by high-fat $\operatorname{diet}(\mathrm{HFD})$. Moreover, the possible anti-hyperlipidemic mechanisms involving inhibiting the cholesterol absorption would also be discussed.

\section{Methods}

\section{Chemicals and reagents}

Berberine was purchased from Kangdele Pharmaceutical Enterprise (Chengdu, China), while Evodiamine was purchased from Chengdu ruiteen science and Technology Co., Ltd., and dissolved in $0.5 \%(v / v)$ Carboxymethyl cellulose. Ezetimibe was supplied by Pfizer Pharmaceuticals Ltd. Commercial kits for testing plasma total cholesterol (TC), triglyceride (TG), hepatic TC, and hepatic TG was supplied by Applygen T Gene Technology Co. Ltd. The kits for measurement of low-density lipoprotein cholesterol (LDL-C) and high-density lipoprotein cholesterol (HDL-C) were supplied by Jiancheng Bioengineering Institute (Nanjing, Jiangsu, China).

\section{Animals}

A total of 60 male Sprague-Dawley (SD) rats (10 weeks old, body weight: $200 \pm 20$ g) were obtained from Chengdu Dashuo experimental animal Co. Ltd. (Certificate no. SCXK (Sichuan) 2013-24). All animals were housed 5 per cage under the controlled conditions (temperature 22 $\pm 2{ }^{\circ} \mathrm{C}$, relative humidity $50 \% \pm 5 \%$, and $12 \mathrm{~h}$ light/dark cycle), and had access to food and water ad libitum. All the rats were fed with the standard solid food as acclimatization for 7 days prior to the experiments. All of the animal protocols were performed in strict accordance with the guidelines for the care and use of laboratory animals established by the Animal Ethical Committee of Chengdu University of Traditional Chinese Medicine (CDUTCM). All surgery was executed under sodium pentobarbital anesthesia, which kept the experimental damage at a minimum.

\section{Experimental design and supplementation}

The 60 rats were randomly assigned to two groups: a normal control group (CON) of 10 was fed with a normal laboratory diet, while 50 rats were fed with HFD for 4 weeks to induce hyperlipidemia. The composition of HFD followed the method of Zhang, Q and co-workers, which contained a normal diet supplemented with $1 \%$ cholesterol, $10 \%$ lard, $5 \%$ yolk powder, $0.2 \%$ propylthiouracil and $1 \%$ sodium tauroglycocholate [20]. After 4 weeks of feeding, the 50 HFD induced rats were subdivided into five groups: a group of 10 (HFD) was treated with sodium carboxymethyl cellulose (CMC; $0.5 \%)$; the other four groups were treated with ezetimibe (EZ) $(10 \mathrm{mg} / \mathrm{kg}$ b.w.), berberine (BB) $(72.6 \mathrm{mg} / \mathrm{kg}$ b.w.), evodiamine (EV) (16.6 mg/kg b.w.), and combination of berberine and evodiamine (BB + EV) $(89.2 \mathrm{mg} / \mathrm{kg}$ b.w.). The berberine, evodiamine, and their combination were dissolved in $0.5 \%$ CMC. These compounds were administrated to rats by gavage at a dose of $10 \mathrm{ml} / \mathrm{kg}$ body weight for four weeks fed with HFD.

\section{Sample collection and preparation}

During the experiment of 8 weeks, blood sample of each rat was collected from the tail vein under fasting conditions once a week at 9:00 AM, and animal weight of the rats was measured weekly. Serum was separated by centrifugation for $5 \mathrm{~min}$ at $3500 \mathrm{rpm}$, stored at $-80{ }^{\circ} \mathrm{C}$. At the end of the experimental period, the animals were fasted for $12 \mathrm{~h}$ and then sacrificed after 10\% chloral hydrate anesthesia. Cardiac puncture was instantly executed to collect the blood. Livers were excised, weighted and divided into two parts: One part was fixed in $10 \%$ neutral buffered formalin for the histopathological detections, the other part was stored at $-80{ }^{\circ} \mathrm{C}$ for further biochemical studies. Small intestine was resected in a small section (about 8-10 cm) from each rat, stored at $-80{ }^{\circ} \mathrm{C}$ for immunohistochemical analysis. $10 \% \mathrm{w} / \mathrm{v}$ 
homogenate compound was prepared with $0.1 \mathrm{~g}$ of liver tissue in $1 \mathrm{ml}$ of $0.1 \mathrm{M}$ phosphate buffer ( $\mathrm{pH} 7.4$ ), then centrifuged at $4000 \mathrm{rpm}$ for $10 \mathrm{~min}$, kept frozen at $-80{ }^{\circ} \mathrm{C}$ for biochemical assays.

\section{Lipid profile analysis}

The levels of TC, TG, LDL-C, and HDL-C in rat serum were measured by commercial reagent kits respectively, according to the standards and protocols provided by the manufacturer. The liver tissue was homogenized with phosphate buffer ( $\mathrm{pH}$ 7.4) as density of $10 \%$ and centrifuged at $4000 \mathrm{rpm}$ for $10 \mathrm{~min}$. The levels of total cholesterol and triglycerides in the liver were determined by enzymatic-colorimetric assay kits respectively. All the procedures were following the instructions offered by the kits.

\section{Histopathological examination}

The liver tissues were fixed in 10\% neutral paraformaldehyde overnight. Then, the samples were dehydrated through graded ethanol series, made transparent with xylene, embedded with paraffin. The sections were cut into 4-8 $\mu \mathrm{m}$ slices and stained with hematoxylin and eosin staining (H\&E staining). There were 5 slices for each animal used for pathological observation. The sections were observed at $400 \times$ magnification under light microscope.

\section{Gas chromatography analysis}

Concentrations of $\beta$-sitosterol (BS) and cholesterol in serum were measured using Gas Chromatograph (GC) technique. The plasma samples preparation were operated by mixing $0.1 \mathrm{ml}$ of serum sample with $0.1 \mathrm{ml}$ of internal standard, $1.0 \mathrm{ml}$ of absolute ethyl alcohol, and $1.0 \mathrm{~mL}$ of $8.9 \mathrm{~mol} / \mathrm{L} \mathrm{KOH}$. The solution was mixed for $15 \mathrm{~s}$ and saponified in $60{ }^{\circ} \mathrm{C}$ for $60 \mathrm{~min}$, extracted with hexyl hydride. The supernatant was transferred to a $25 \mathrm{~mL}$ volumetric flask and evaporated to dryness at $40{ }^{\circ} \mathrm{C}$. $600 \mu \mathrm{l}$ of silylating reagent (TMCS / HMDS/ Pyridine $(3: 1: 9, v / v))$ were added to the solution to catalyze the reaction which proceeded for $30 \mathrm{~min}$ at $60{ }^{\circ} \mathrm{C}$. After evaporated to dryness, the reaction system was added with $1 \mathrm{ml}$ of hexyl hydride. Then, the solution was mixed and centrifuged at $12000 \times \mathrm{g}$ at $4{ }^{\circ} \mathrm{C}$ for $1 \mathrm{~min}$. Liquid supernatant was were separated for Gas chromatography analysis. One microliter of prepared sample was injected into a 7890A Gas Chromatograph (Agilent, USA). Chromatographic separation was carried on a HP-5 quartz capillary column $(30 \mathrm{~m} \times 0.32 \mathrm{~mm} \times 0.25 \mu \mathrm{m})$, and high purity nitrogen (99.99\%) was applied as carrier gas. The temperature of Hydrogen flame ionization detector (FID) was set to $290{ }^{\circ} \mathrm{C}$. The temperature and pressure of injection port were controlled at $290{ }^{\circ} \mathrm{C}$ and $18 \mathrm{psi}$, respectively, while no spit mode administrated. The initial temperature of column was kept at $180{ }^{\circ} \mathrm{C}$ for $3 \mathrm{~min}$. The temperature was following increased up to $260{ }^{\circ} \mathrm{C}$ at a rate of $15{ }^{\circ} \mathrm{C} / \mathrm{min}$, and then elevated to $300{ }^{\circ} \mathrm{C}$ at a rate of $5{ }^{\circ} \mathrm{C} / \mathrm{min}$ and held for $10 \mathrm{~min}$. Plant sterols were identified by the gas chromatography peaks in contrast with the standards, which were measued as mg fatty acid per $\mathrm{ml}$ plasma volume.

\section{Immunohistochemistry assay}

The intestinal samples were washed by PBS rapidly and fixed with $4 \%$ paraformaldehyde overnight at $4{ }^{\circ} \mathrm{C}$. With accomplishment of $4 \mu \mathrm{m}$ paraffin slices, the sections were deparaffinized with xylene and rehydrated through gradient ethanol, treated with $3 \%$ hydrogen peroxide methanol for 10 min which were used to inactivate endogenous peroxidase. Washed with PBS for 3 times, tissue sections were subjected to $10 \mathrm{mM}$ sodium citrate buffer ( $\mathrm{pH}$ 6.0) and heated in microwave oven with middle power for $10 \mathrm{~min}$. After blocked in goat serum for $20 \mathrm{~min}$ at room temperature, the sections were incubated with primary polyclonal rabbit anti- ACAT2, antiNPC1L1, or anti- Apo B, respectively, at $4{ }^{\circ} \mathrm{C}$ for one night. Subsequently, sections were incubated in goat anti-rabbit $\operatorname{IgG}$ for $30 \mathrm{~min}$ at $37{ }^{\circ} \mathrm{C}$ before permeabilization in PBS. Diaminobenzidine was applied for chromogenic reaction for $2 \mathrm{~min}$ at the room temperature. Then slides were treated with Light Mayer hematoxylin for counterstaining, and then dehydrated by graded concentration of ethanol, finally covered with Neutral Canada gum. Images were captured using an BA400 Digital confocal microscope. The integrated optical density (IOD) of ACAT2, NPC1L1, and Apo B expressions were measured by Image-Pro Plus 6.0 software (Media Cybernetics, Siver Spring, MD, U.S.A.), meanwhile the images were managed with Adobe Photoshop 8.0.

\section{Statistical analysis}

All experimental data were expressed as mean \pm standard deviation (S.D.). Statistical analyses were evaluated by one-way analysis of variance (ANOVA) and twotailed Student's t tests with SPSS19.0 software. A level of $P$-value $<0.05$ was considered statistically significant. All the figures were carried out by Graph Pad Prism 6.0 software.

\section{Results}

\section{Animal and organ weights}

During the experimental period, body weight and food intake declined remarkably in the HFD-fed groups compared with those in CON group $(P<0.01)$. However, no significant difference was showed among the groups administrated by different treatments in body weight and food intake $(P>0.05)$ (Fig. 1a and b). Liver weight index 


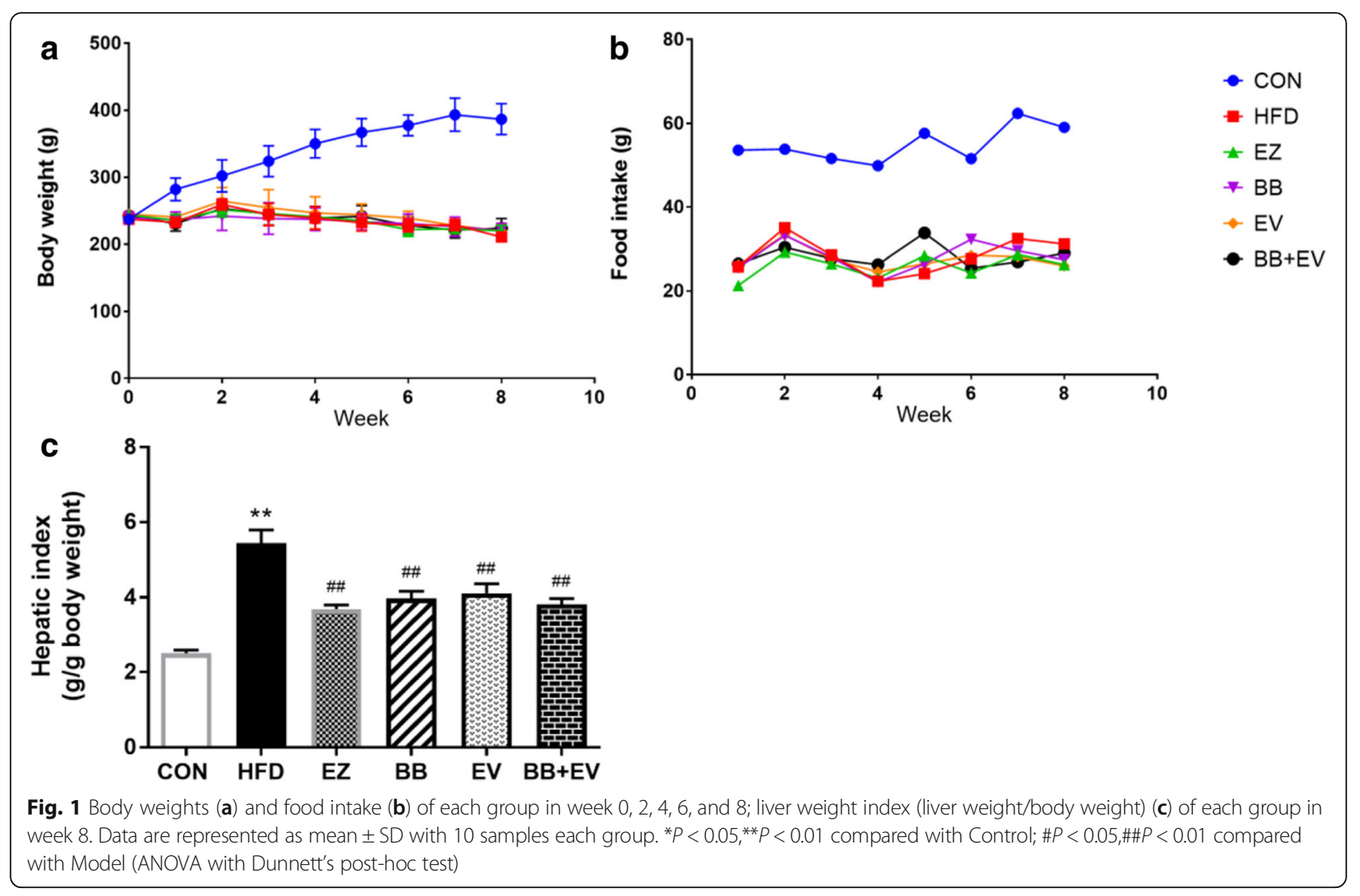

was detected as the ratio of liver weight to body weight to properly compare individual variation on liver pathology. Compared with CON, HFD group showed remarkable increase on liver weight index, which were significantly reduced by $\mathrm{BB}, \mathrm{EV}$, and $\mathrm{BB}+\mathrm{EV}$ $(P<0.01)$ (Fig. 1c).

\section{Serum lipid profiles}

The level of serum TC, TG, HDL-C, and LDL-C in hyperlipidemic rats at 4th and 8th week were showed in Fig. 2-3. By comparison with CON group, meaningful elevation of serum TC and LDL-C was observed in the HFD-fed rats in week $4(P<0.05$ or $P<0.01)$ (Fig. 2a, c). But the concentration of serum TG and HDL-C did not show significant difference when rats were fed with HFD $(P>0.05)$ (Fig. 2b, d).

After the continuation of HFD supplementation and treatment by tested drugs for 4 weeks, remarkable reductions were observed in hyperlipidemic rats treated by different drugs in week 8 (Fig.3).The serum levels of TC, $\mathrm{TG}$, and LDL-C were significantly decreased in group $\mathrm{BB}, \mathrm{EV}$, and $\mathrm{BB}+\mathrm{EV}$ compared with those in HFD group in week $8(P<0.05$ or $P<0.01)$ (Fig. 3a, b, c). Only plasma HDL-C concentration showed a difference between HFD group and other drug treated groups. The administration of $\mathrm{BB}$ and $\mathrm{EV}$ for 4 weeks resulted in notable decreases in serum HDL-C levels $(P<0.05$ or $P<0.01$ ), while there was no statistical significance on LDL-C level when compared with the HFD group (Fig. 3d).

\section{Lipid content of the liver}

Hepatic cholesterol and triglycerides were measured at the end of the 8-weeks experimental period to analyze the effect of $\mathrm{BB}$ and $\mathrm{EV}$ on tissue lipid content. Compared with CON group, the level of hepatic TC were significantly increased in hyperlipidemic rats induced by HFD $(P<0.01)$. Hepatic TC content in BB + EV group were remarkable decreased by $26 \%$ compared with the HFD group $(P<0.05)$. But no noticeably significant was showed when rats were administrated only with $\mathrm{BB}$ or EV (Fig. 4a). Treatment with $\mathrm{BB}$ and BB + EV tended to reduce hepatic triglycerides levels, although just in the edge of significance $(P>0.05)$ (Fig. $4 \mathrm{~b})$.

\section{Histological analyses of the liver}

After supplementation of HFD for eight weeks, liver structures and the degree of lipids accumulation were characterized by hematoxylin-eosin (H\&E) staining on liver tissues of rats with hyperlipidemia. As showed in Fig. 5, the rats in CON group showed a well- structured liver. HFD rats manifested pathological changes of the 

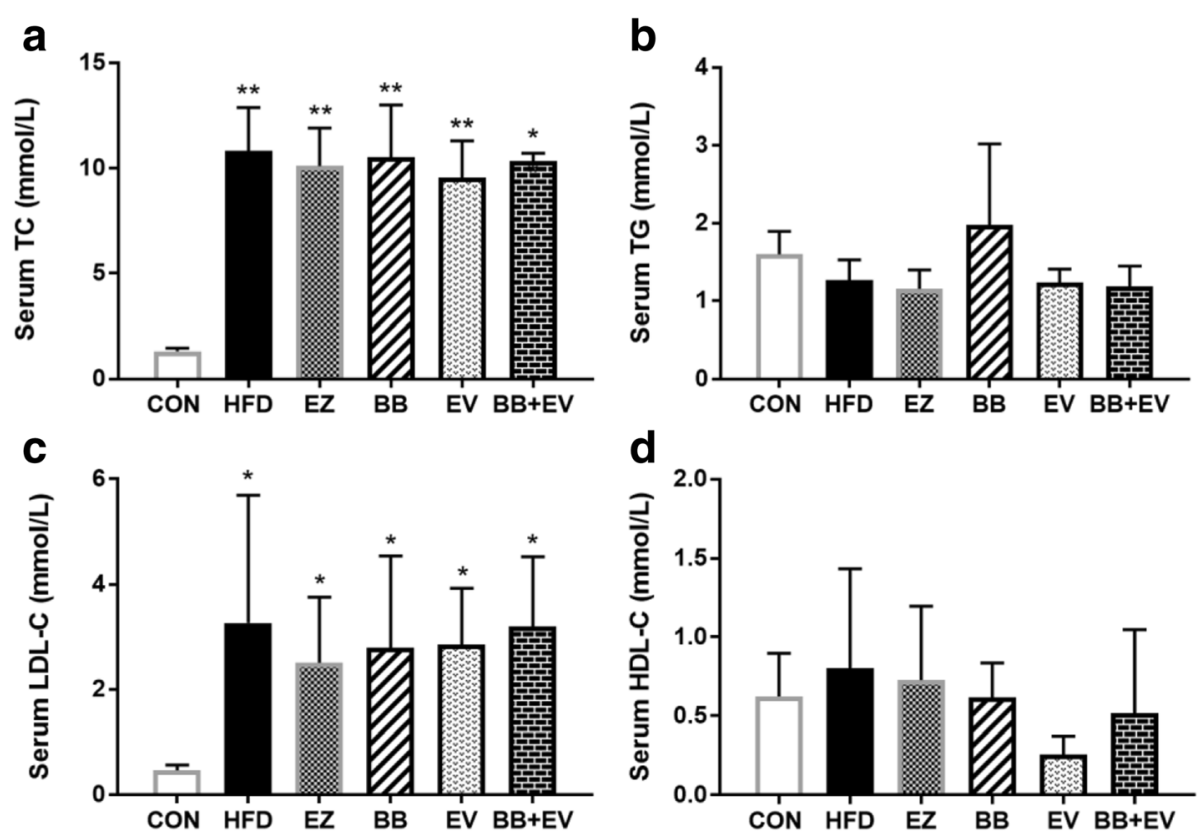

Fig. 2 Serum TC (a), serum TG (b), serum LDL-C (c), and serum HDL-C (d) in hyperlipidemic rats in week 4. Data are represented as mean \pm SD with 10 samples each group. ${ }^{*} P<0.05$, ${ }^{* *} P<0.01$ compared with Control; $\# P<0.05$,\#\#P<0.01 compared with Model (ANOVA with Dunnett's post-hoc test)

liver manifested swollen hepatocytes coupled with hepatocytes steatosis in the form of increasing degree of vacuolar degeneration. By comparison of HFD group, these changes could be obviously alleviated by EZ, BB, and $\mathrm{BB}+\mathrm{EV}$ (Fig. 5). HE staining indicated that fatty liver changes accompanied with neutrophils infiltrating into acini area were resulted from HFD, which could be obviously inhibited by EZ, BB, and BB + EV.

\section{Absorption markers of cholesterol}

Plasma concentrations of BS and cholesterol measured by $\mathrm{GC}$ analysis in hyperlipidemic rats were showed in

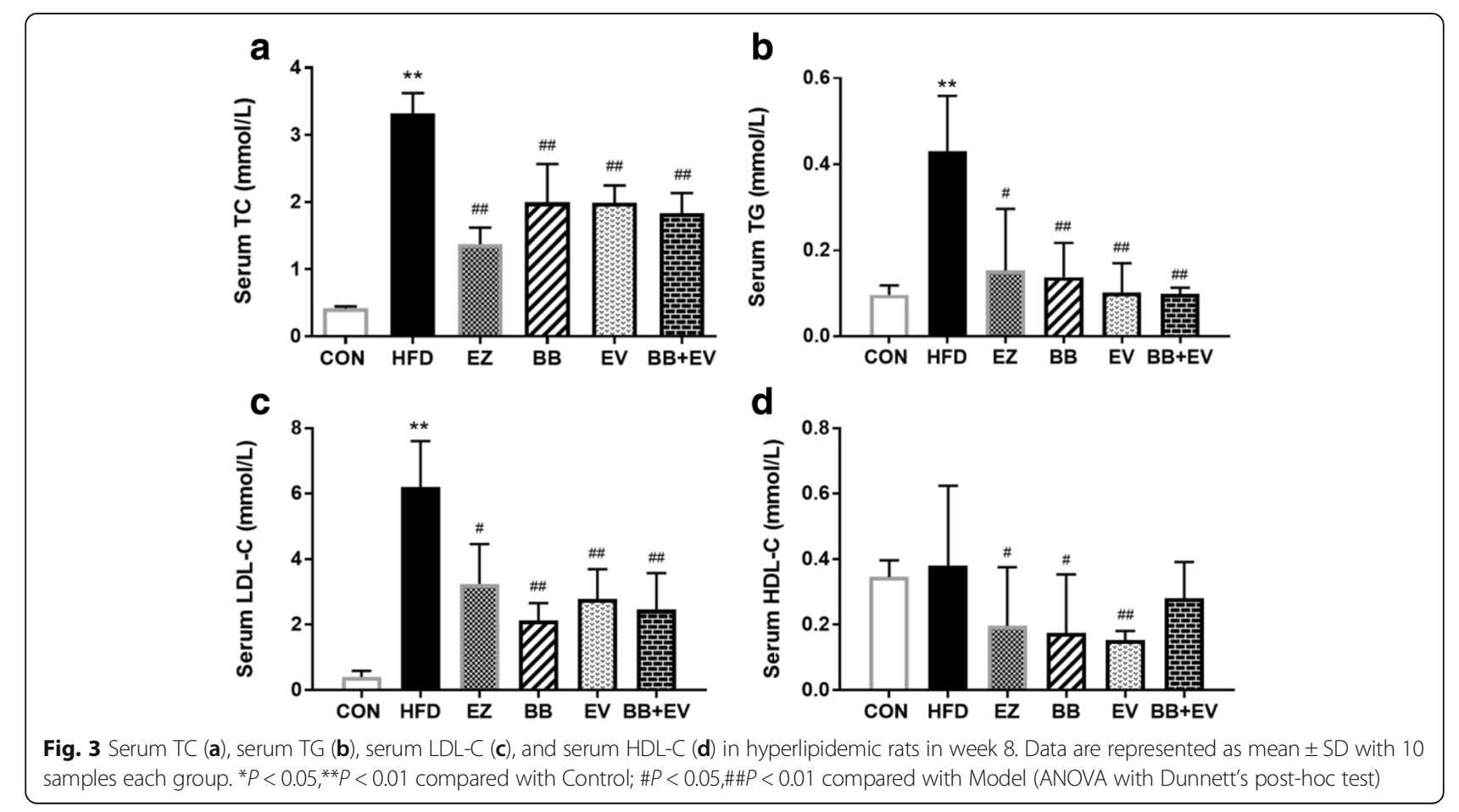



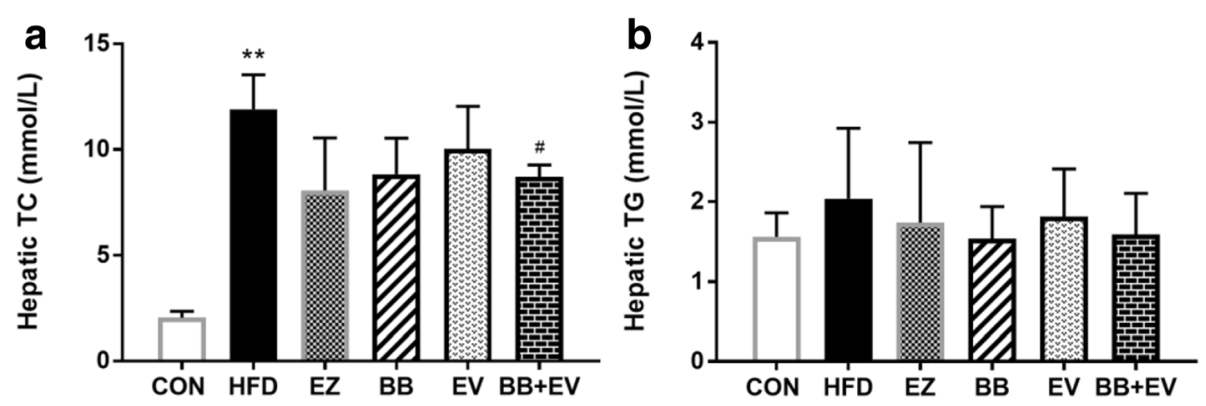

Fig. 4 Hepatic TC (a) and hepatic TG (b) content of hyperlipidemic rat in week 8. Data are represented as mean \pm SD with 10 samples each group. ${ }^{*} P<0.05$, ${ }^{* *} P<0.01$ compared with Control; $\# P<0.05, \# \# P<0.01$ compared with Model (ANOVA with Dunnett's post-hoc test)

Fig. 6. By the continuation of 8 weeks supplementation with the HFD, serum levels of BS and cholesterol were significantly elevated in the HFD group compared with the CON group $(P<0.01)$. After 4 weeks of treatment with the therapies, serum contents of BS and cholesterol were rapidly decreased in group $\mathrm{BB}, \mathrm{EV}$, and $\mathrm{BB}+$ EV $(P<0.01)$ (Fig. 6a, b). Additionally, the ratio of $B S$ to cholesterol developed a significant decrease in HFD group, which was alleviated by $\mathrm{BB}$ and $\mathrm{BB}+\mathrm{EV}$ $(P<0.05)$ (Fig. 6c).

\section{Immunohistochemistry}

The immunohistochemical staining of intestine tissues in hyperlipidemic rats were shown in Fig. 7. Positive cells expressed as brown or yellow particle, while the negative cells showed blue stained nucleus. ACAT2, NPC1L1, and ApoB48 positive reactions were mainly distributed in the small intestine mucosa epithelium according to the staining results (Fig. $7 \mathrm{~d}$, e, and f). Meanwhile, the integrated optical density (IOD) of ACbAT2, NPC1L1, and ApoB48 expressions were calculated to evaluate the effect of berberine combined with evodiamine on cholesterol absorption in intestine.
Compared with the CON group, HFD increased the expressions of ACAT2, NPC1L1, and ApoB48 significantly. Furthermore, berberine combined with evodiamine could remarkably decrease the expressions of intestine ACAT2, NPC1L1, and ApoB48 compared with HFD group $(P<0.05)$ (Fig. $7 \mathrm{a}, \mathrm{b}$, and $\mathrm{c}$ ). Treatment with $\mathrm{BB}$ alone had the trend to decrease the expression of intestinal NPC1L1 protein, even though barely failed to attain statistical significance $(P>0.05)$ (Fig. 7a).

\section{Discussion}

Hyperlipidemia presents as a principal factor of accelerating the progression of Cardiovascular disease (CVD) which results in morbidity and mortality in developed countries [21]. Treatments of lipid lowering is wellrecognized as a notable strategy by all the health care organizations around the world for prevention of CVD [22]. A large number of natural plant products found in traditional Chinese medicine (TCM) have been proved to possess lipid-lowing activities with fewer side effects, which draw great attention worldwide [23]. These findings have enhanced the searching for the alternative drugs with fewer adverse effects to prevent the risk of
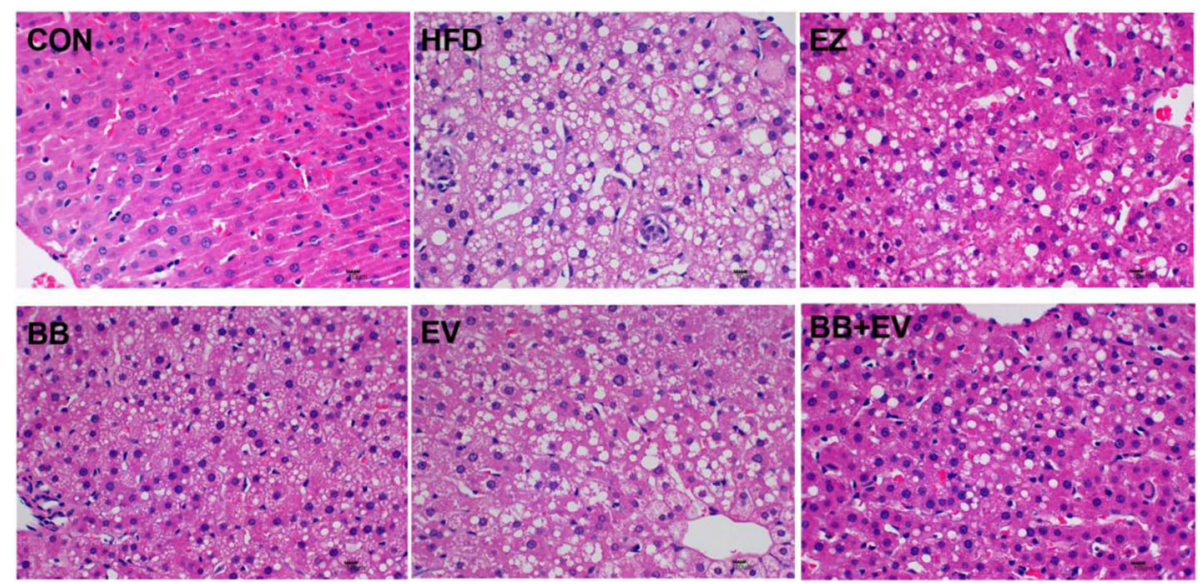

Fig. 5 Effect of berberine and evodiamine co-administration on the pathology of liver tissues in hyperlipidemic rats. Specimens were photographed by light microscopy. ( $H$ and E staining, magnification: $\times 400, n=6$ ) 

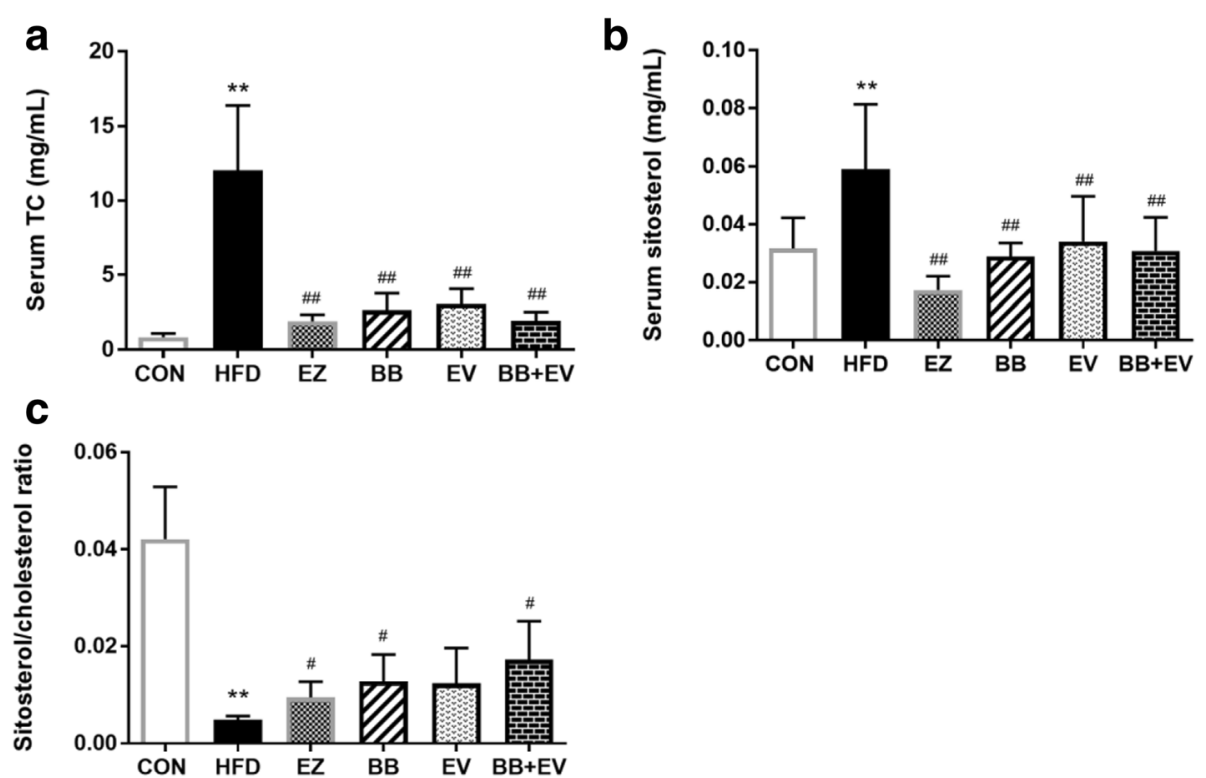

Fig. 6 Serum total cholesterol (a), serum sistosterol (b), and the ratio of sistosterol by cholesterol (c) measured by gas chromatography in hyperlipidemic rat. Data are represented as mean \pm SD with 10 samples each group. ${ }^{*} P<0.05$, ${ }^{*} P<0.01$ compared with Control; \#P<0.05,\#\#P<0.01 compared with Model (ANOVA with Dunnett's post-hoc test)

CVD. The combination of berberine and evodiamine is from a two-herb Chinese medicinal formula composed of Coptis chinensis and Evodia rutaecarpa, which has been widely used to treat multiple diseases in terms of different ratios [24]. Berberine is the active ingredient of the traditional herbal Coptidis Rhizoma which has been investigated in many directions, including antiinflammatory, anti-cancer,anti-microbial, as well as cardiovascular fields [7-10]. Evodiamine is a naturally indoloquinazoline alkaloid isolated from Evodia rutaecarpa, attracting close attentions with its anti-cancer, anti-inflammation, and anti-obesity effects [13-17]. Many researches have indicated that both of berberine and evodiamine exerted on regulation of lipid metabolism $[18,25]$, but whether they have a synergistic effect on preventing hyperlipidemia when combined together remains completely unclear.

In the present study, the effect of the berberine combined with evodiamine was compared to the effects of a single herb on the parameters of hyperlipidemia in HFD-fed rats. The current study demonstrated that berberine, evodiamine, and their combinations could obviously reduce the serum lipid hyperlipidemic rats induced by HFD. Moreover, the combination of berberine and evodiamine notably decreased the content of TC in serum and liver, which showed best lipid-lowing effect compared with the either drug alone. The results appeared to be show a synergistic action of berberine and evodiamine as the effect on serum TC reduction. The underlying mechanism was exerted by the characterization of key proteins involved in the process of cholesterol absorption.
Diet habits play a significant role in the development of cholesterol metabolism. It is believed that the consumption of high fat diet which contains of saturated fats is the major risk factor for development of obesity and metabolic syndrome [26]. In this study, the hyperlipidemic animal model was successfully established in rats by the induction of high-fat, high-cholesterol diet, as evidenced by increased lipid parameters in serum and liver. In our study, a dramatic increase in serum lipid levels of TC, TG, LDL-C, and hepatic TC levels was observed in hyperlipidemic rats induced with HFD in 4 weeks (Fig. 2). After administration with berberine, evodiamine, and their combination, the elevated serum levels of TC, TG, and LDL-C were significantly decreased at eight week (Fig. 3). Meanwhile, cholesterol content in liver was obviously decreased after the supplementation of BB $+E V$. Notably, treatment with $\mathrm{BB}+\mathrm{EV}$ showed the best effect on normalizing the serum TC, TG, and hepatic TC concentrations in 8 weeks, which appeared superior to either herb alone. However, $\mathrm{BB}$ and $\mathrm{BB}+\mathrm{EV}$ did not develop a significant effect on body weight compared with HFD group (Fig. 1a, b), which agreed with the study of Yanwen Wang et al. [12], who found that berberine did not show a significant effect on body weight. Berberine was conformed to possess obvious cholesterollowering effect on serum TC, LDL-C, or non HDL cholesterol (non HDL-C) in plenty of researches [12, 27-29]. Evodiamine was reported to have effect on lipid metabolism by regulation the key genes in fat synthesis [18]. The reductions of liver cholesterol might be a result of 

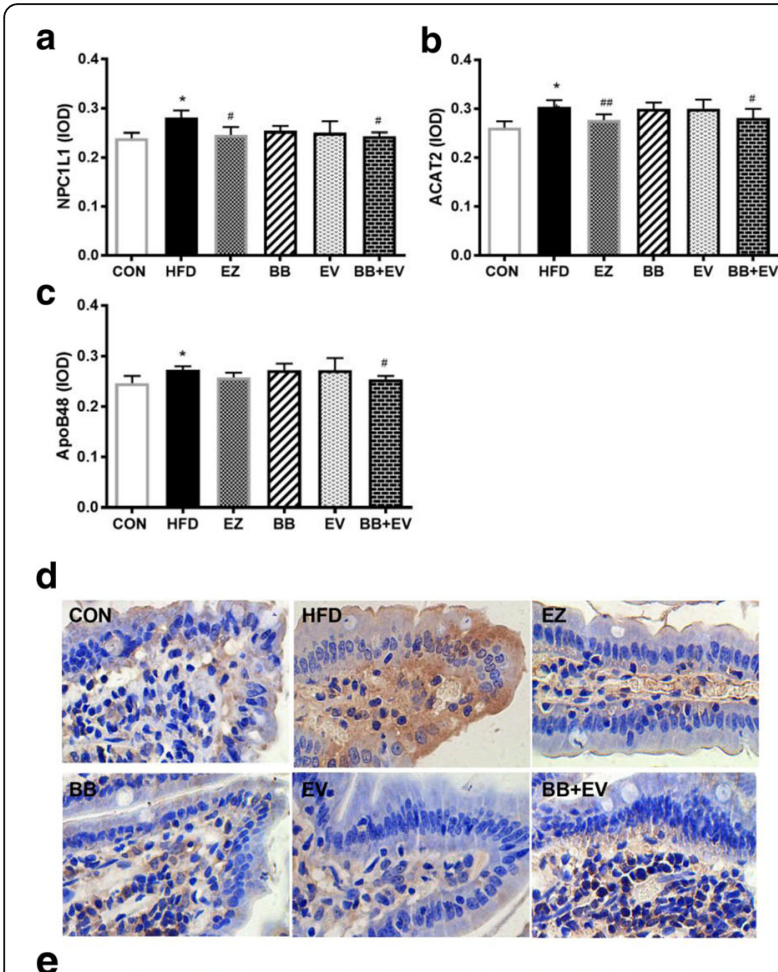

e

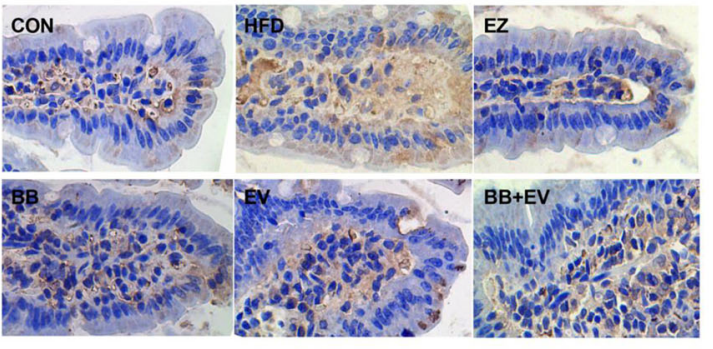

f

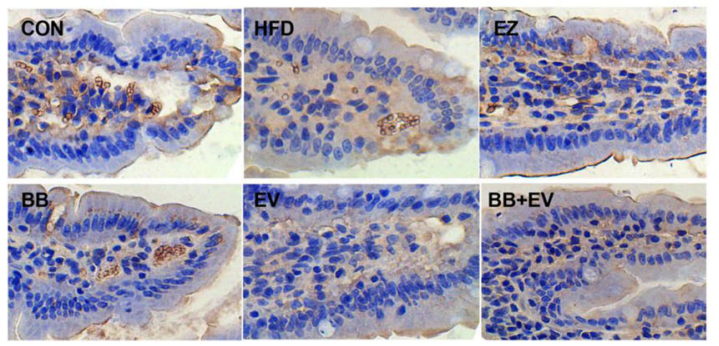

Fig. 7 Integrated optical density (IOD) of NPC1L1(a), ACAT2 (b), and ApoB48 (c) in HFD induced hyperlipidemic rats ( $n=6$, mean \pm SD). ${ }^{*} P<0.05$, ${ }^{* *} P<0.01$ compared with Control; $\# P<0.05$, \#\#P<0.01 compared with Model (ANOVA with Dunnett's post-hoc test). Histopathological examination of NPC1L1 (d), ACAT2 (e), and ApoB48 (f) in intestine tissue of hyperlipidemic rats in different experiment groups $(\times 400$ magnifications, $n=6$ )

decreased cholesterol input arise from the inhibition of intestinal cholesterol absorption [30]. Our research showed that the combination of berberine and evodiamine had the best lipid-lowing effect on lipid profile in serum and liver, compared to either drug alone. Therefore, we speculate that the berberine combined with evodiamine had a synergistic effect on reducing cholesterol in the plasma and liver by inhibiting intestinal cholesterol absorption.

Large numbers of studies have demonstrated the hypolipiemic properties of berberine and evodiamine by animal and clinical researches. Kong et al. [31] has been reported that $1000 \mathrm{mg} /$ day berberine for three months could significantly lower total cholesterol and LDLcholesterol by $29 \%$ and $25 \%$. Another study has showed that evodiamine as a supplement comprising 0.02, 0.04, and $0.06 \%$ of the diet fed to mice for 4 weeks could influence lipid metabolism in time and dose-dependent manners [18]. However, minimal information on the adverse drug reactions of berberine and evodiamine supplementation is available. Some studies have demonstrated that the maternal toxicity lowest observed adverse effect levels of berberine were $531 \mathrm{mg} / \mathrm{kg} /$ day and $841 \mathrm{mg} / \mathrm{kg} /$ day in rats and mice [32], and berberine could present certain side effects in hypervagotonic people [33]. The incidence of toxic adverse effect is connected with the doses of berberine, which showed that the dose of berberine increase lead to the rising risk of toxic side effect [34]. In our current study, the doses of berberine and evodiamine were $72.6 \mathrm{mg} / \mathrm{kg}$ and $16.6 \mathrm{mg} / \mathrm{kg}$, which were equal to $5 \mathrm{~g} /$ day of the human dose, much lower than that used in previous studies. Whether the supplementation of berberine combined with evodiamine has any side effects for a long time as a treatment on hyperlipidemia needs a further study in the future.

Hyperlipidmia is characterized as the increased serum lipid index especially serum total cholesterol and LDL cholesterol levels, which results in coronary morbidity and mortality. The previous researches suggested that the ratios of serum cholestanol and plant sterols to cholesterol were the markers of cholesterol absorption, which reliably depicted the cholesterol absorption efficiency. Non-cholesterol sterols which include cholestanol and plant sterols, are regarded to be significant markers of cholesterol absorption [35]. Thongtang et al. [36] found that the ratio of $ß$-sitosterol to total cholesterol was positively related to absorption activity of cholesterol. In this study, we found that supplementation with HFD increased the level of $ß$-sitosterol and $\beta$-sitosterol/cholesterol ratio, which were attenuated by berberine, evodiamine, and their combination, suggesting that berberine, evodiamine, and their combination could successfully decrease the markers of cholesterol absorption, ultimately inhibiting absorption of cholesterol. Notably, treatment with the combination of berberine and evodiamine showed remarkable reduction in $\beta$-sitosterol by $48.2 \%$ compared with the HFD group. Although berberine alone also inhibited the cholesterol absorption 
marker, this effect was intensified when it was combined with evodiamine. Therefore, we hypothesized that the hyperlipidemic ability of co-administration of berberine and evodiamine may be associated with their effects on cholesterol absorption.

Absorption of cholesterol is a significant physiological process involved in maintaining lipid homeostasis [4]. Cholesterol absorption occurs mainly in the small intestine and proximal jejunum,which is regulated by a number of proteins. Niemann-Pick C1 like1 (NPC1L1) protein and Acyl-CoA cholesterol acyltransferase 2 (ACAT2) are essential factors on regulating the intestinal absorption of cholesterol. Cholesterol absorption starts with cholesterol uptake by enterocyte. Apart from the passive penetration, free cholesterol which is hydrolyzed from dietary cholesterol esters and biliary sources are transported actively through NPC1L1 from the lumen into enterocytes [37]. Subsequently, free cholesterol is converted to cholesteryl ester (CE), packed into chylomicrons $(\mathrm{CM})$ and then transferring into the lymphatics. This process is catalyzed by ACAT2, which is a key cholesterol esterifying enzyme for chylomicrons biosynthesis from cholesteryl esters [38]. After the general circulation, cholesterol is ultimately delivered to the liver as the form of CM. Our study showed that the coadministration of berberine and evodiamine favorably inhibit the expressions of NPC1L1 and ACAT2 accompany by the reductions of serum cholesterol levels, which suggested that the hyperlipidemic effect of the combination might be attributed to the down-regulation of NPC1L1 and ACAT2 in HFD-fed rats. Elimination of excessive cholesterol contained mainly two ways, which included firstly, combining the intracellular cholesterol into cholesterol esters in a way activation of ACAT2. Second, excess cholesterol was converted to bile acids in the liver, finally eliminated into feces [39]. The second possible mechanism for the cholesterol-lowering activity of berberine and evodiamine supplementation was mediated by increasing the bile acid synthesis. We plan to investigate the effect of combination of berberine and evodiamine on bile acid profile in the future study.

Apolipoprotein B is an important plasma particle which is the formation of chylomicrons and the other cholesterol transport molecules as low-density lipoprotein (LDL) and very-low-density lipoprotein (VLDL) for cholesterol transportation between liver and peripheral tissues [40]. ApoB48 is the transport molecules essential for $\mathrm{CM}$, responsible for packing the dietary fats to form CMs for transportation [41]. In fact, ApoB48 was accepted as a vital element involved in lipid metabolism, even a risk factor for CVD [42]. In line with previous study, the current study found that the expression of ApoB48 in intestine was importantly elevated in HFD group by comparison with the $\mathrm{CON}$ group. Interestingly, berberine combined with evodiamine significantly decreased intestinal ApoB48 expression in HFD-fed rats. These results indicated that the combination of berberine and evodiamine exhibited the lipid-lowing effect partly on inhibiting the intestinal absorption of dietary fat by reducing ApoB48 overproduction.

\section{Conclusion}

In conclusion, our study indicated that berberine, evodiamine, and their co-administration can lower blood cholesterol levels in hyperlipidemic rat induced by high fat diet. Additionally, evodiamine functions as an enhancer of berberine to promote the lipid-lowing effect on serum cholesterol in HFD-induced rats, and the potential mechanism appears to involve inhibiting the expression of ACAT2, NPC1L1, and ApoB48 to depress the cholesterol absorption. Thus, our outcomes demonstrated that the combination of berberine and evodiamine possessed potential lipid-decreasing effect, and further researches on berberine combined with evodiamine deserve to be carried out to provide new insight into maintaining healthy lipid profiles.

\section{Abbreviations \\ ACAT2: Acyl-CoA cholesterol acyltransferase 2; ApoB48: Apolipoprotein B48.; BB: Berberine; BS: ß- Sitostero; CE: Cholesteryl ester; CM: Chylomicrons; CMC: Sodium carboxymethyl cellulose; CON: Control; CVD: Cardiovascular disease; EV: Evodiamine; EZ: Ezetimibe; FID: Flame ionization detector; GC: Gas chromatography; HDL-C: High density lipoprotein-cholesterol; HFD: High fat diet; HMDS: Hexamethyl Disilazane; LDL-C: Low density lipoprotein-cholesterol; LDLR: Low density lipoprotein receptor; NPC1L1: Niemann-Pick C1 like1; TC: Total cholesterol; TCM: Traditional Chinese Medicine; TG: Triglycerides; TMCS: Tri Methyl Chloro Silane; VLDL: Very-low-density lipoprotein}

\section{Acknowledgements}

We appreciate Fang Zeng and Yunhui Chen for the assistance on manuscript revision. And we also thank Qian Wu, Ting Pan, Hang Zheng and Lixia Qin for their technical support on data collections.

\section{Funding}

This work was supported by National Natural Science Foundation of China (No. 81373545 and No. 81703844), and Education Department of Sichuan Province (No.17ZB0147).

Availability of data and materials

All data used during the current study are available from the corresponding author on reasonable request.

\section{Authors' contributions}

XZ performed the research, carried out the data collection and analysis, and drafted the manuscript. FYR contributed to the Gas chromatography analysis and manuscript revision. HW conducted the study, and participated in data analysis. LYL participated in the study coordination, and helped to revise the manuscript. TS and SJX provided experimental concepts and design, offered scientific direction, and reviewed the manuscript. JPW, JYR and HFN performed the assessment of lipid profile levels, and participated in data collection. All authors participated in the manuscript preparation, read and approved the final manuscript

\section{Ethics approval}

The animal protocol was approved by the Animal Ethical Committee of Chengdu University of Traditional Chinese Medicine (Sichuan, China). 


\section{Consent for publication}

Not applicable.

\section{Competing interests}

The authors state that they have no competing interests.

\section{Publisher's Note}

Springer Nature remains neutral with regard to jurisdictional claims in published maps and institutional affiliations.

\section{Author details}

'School of Basic Medicine, Chengdu University of Traditional Chinese Medicine, Chengdu 611137, China. ${ }^{2}$ Antibiotics Research and Re-evaluation Key Laboratory of Sichuan Province, Sichuan Industrial Institute of Antibiotics, Chengdu University, Chengdu 610052, China. ${ }^{3}$ School of International Education, Chengdu University of Traditional Chinese Medicine, Chengdu 611137, China. ${ }^{4}$ School of Pharmacy, Chengdu University of Traditional Chinese Medicine, Chengdu 611137, China.

\section{Received: 24 August 2017 Accepted: 28 November 2017}

Published online: 11 December 2017

\section{References}

1. Xiao C, et al. Pharmacological targeting of the Atherogenic dyslipidemia complex: the next frontier in CVD prevention beyond lowering LDL cholesterol. Diabetes. 2016;65(7):1767-78.

2. Watts GF, Karpe F. Triglycerides and atherogenic dyslipidaemia: extending treatment beyond statins in the high-risk cardiovascular patient. Heart. 2011; 97(5):350-6.

3. Okerson T, et al. Effect of 2013 ACC/AHA blood cholesterol guidelines on statin treatment patterns and low-density lipoprotein cholesterol in atherosclerotic cardiovascular disease patients. J Am Heart Assoc. 2017;6(3)

4. Li $Y$, et al. Adipose tissue regulates hepatic cholesterol metabolism via adiponectin. Life Sci. 2014;118(1):27-33.

5. Rudel LL, Lee RG, Parini P. ACAT2 is a target for treatment of coronary heart disease associated with hypercholesterolemia. Arterioscler Thromb Vasc Biol. 2005;25(6):1112-8.

6. Gylling $H$, et al. Polymorphisms in the ABCG5 and ABCG8 genes associate with cholesterol absorption and insulin sensitivity. J Lipid Res. 2004;45(9): 1660-5.

7. Choi $\mathrm{BH}$, et al. Berberine reduces the expression of adipogenic enzymes and inflammatory molecules of 3T3-L1 adipocyte. Exp Mol Med. 2006;38(6): 599-605.

8. Ma W, et al. Berberine inhibits the proliferation and migration of breast cancer ZR-75-30 cells by targeting Ephrin-B2. Phytomedicine. 2017;25:45-51.

9. Wang $X$, et al. Effect of berberine on Staphylococcus Epidermidis biofilm formation. Int J Antimicrob Agents. 2009;34(1):60-6.

10. Dong $\mathrm{H}$, et al. Berberine in the treatment of type 2 diabetes mellitus: a systemic review and meta-analysis. Evid Based Complement Alternat Med. 2012;2012:591654

11. Dong $H$, et al. The effects of berberine on blood lipids: a systemic review and meta-analysis of randomized controlled trials. Planta Med. 2013;79(6): 437-46.

12. Wang $Y$, et al. Berberine decreases cholesterol levels in rats through multiple mechanisms, including inhibition of cholesterol absorption. Metabolism. 2014; 63(9):1167-77.

13. Zhao LC, et al. Evodiamine induces apoptosis and inhibits migration of HCT116 human colorectal cancer cells. Int J Mol Sci. 2015;16(11):27411-21.

14. LV Q, et al. Beneficial effects of evodiamine on P2X(4)-mediated inflammatory injury of human umbilical vein endothelial cells due to high glucose. Int Immunopharmacol. 2015;28(2):1044-9.

15. Chiou WF, et al. The vasorelaxant effect of evodiamine in rat isolated mesenteric arteries: mode of action. Eur J Pharmacol. 1992;215(2-3):277-83.

16. Hu Y, et al. Inhibitory effect and transcriptional impact of berberine and evodiamine on human white preadipocyte differentiation. Fitoterapia. 2010; 81(4):259-68.

17. Bak EJ, et al. Inhibitory effect of evodiamine alone and in combination with rosiglitazone on in vitro adipocyte differentiation and in vivo obesity related to diabetes. Int J Obes. 2010;34(2):250-60.
18. Jiang DF, et al. Long-term effects of evodiamine on expressions of lipogenesis and lipolysis genes in mouse adipose and liver tissues. Genet Mol Res. 2014; 13(1):1038-46.

19. Chao DC, et al. Inhibitory effects of Zuo-Jin-wan and its alkaloidal ingredients on activator protein 1, nuclear factor-kappaB, and cellular transformation in HepG2 cells. Fitoterapia. 2011;82(4):696-703.

20. Zhang Q, et al. Application of GC/MS-based metabonomic profiling in studying the lipid-regulating effects of Ginkgo Biloba extract on diet-induced hyperlipidemia in rats. Acta Pharmacol Sin. 2009;30(12):1674-87.

21. Wang J, et al. Novel biomarkers for cardiovascular risk prediction. J Geriatr Cardiol. 2017:14(2):135-50.

22. Nelson RH. Hyperlipidemia as a risk factor for cardiovascular disease. Prim Care. 2013:40(1):195-211.

23. Li $L$, et al. Herbal drugs against cardiovascular disease: traditional medicine and modern development. Drug Discov Today. 2015;20(9):1074-86.

24. Qian $P$, et al. Pharmacokinetics studies of 12 alkaloids in rat plasma after oral Administration of Zuojin and fan-Zuojin Formulas. Molecules. 2017;22(2)

25. Tan HL, et al. Rhizoma Coptidis: A Potential Cardiovascular Protective Agent. Front Pharmacol. 2016;7:362

26. Kanoski SE, Davidson TL. Western diet consumption and cognitive impairment: links to hippocampal dysfunction and obesity. Physiol Behav. 2011;103(1):59-68.

27. Wang $Y$, et al. Berberine and plant stanols synergistically inhibit cholesterol absorption in hamsters. Atherosclerosis. 2010;209(1):111-7.

28. Kim WS, et al. Berberine improves lipid dysregulation in obesity by controlling central and peripheral AMPK activity. Am J Physiol Endocrinol Metab. 2009; 296(4):E812-9.

29. Jia X, et al. Co-administration of berberine and plant stanols synergistically reduces plasma cholesterol in rats. Atherosclerosis. 2008;201(1):101-7.

30. Hayes $\mathrm{KC}$, et al. Free phytosterols effectively reduce plasma and liver cholesterol in gerbils fed cholesterol. J Nutr. 2002;132(7):1983-8.

31. Kong $W$, et al. Berberine is a novel cholesterol-lowering drug working through a unique mechanism distinct from statins. Nat Med. 2004; 10(12):1344-51.

32. Jahnke GD, et al. Developmental toxicity evaluation of berberine in rats and mice. Birth Defects Res B Dev Reprod Toxicol. 2006;77(3):195-206.

33. Cannillo $M$, et al. Berberine behind the thriller of marked symptomatic bradycardia. World J Cardiol. 2013;5(7):261-4.

34. Lan J, et al. Meta-analysis of the effect and safety of berberine in the treatment of type 2 diabetes mellitus, hyperlipemia and hypertension. J Ethnopharmacol. 2015;161:69-81.

35. <Noncholesterol Sterols and Cholesterol Lowering by.pdf>.

36. Thongtang $\mathrm{N}$, et al. Effects of ezetimibe added to statin therapy on markers of cholesterol absorption and synthesis and LDL-C lowering in hyperlipidemic patients. Atherosclerosis. 2012;225(2):388-96.

37. Altmann SW, et al. Niemann-pick C1 like 1 protein is critical for intestinal cholesterol absorption. Science. 2004;303(5661):1201-4.

38. Lee RG, et al. ACAT2 contributes cholesteryl esters to newly secreted VLDL, whereas LCAT adds cholesteryl ester to LDL in mice. J Lipid Res. 2005;46(6): 1205-12.

39. Jiang $C$, et al. Cholesterol-lowering effects and potential mechanisms of different polar extracts from Cyclocarya paliurus leave in hyperlipidemic mice. J Ethnopharmacol. 2015;176:17-26.

40. Caku A, et al. New insights of altered lipid profile in fragile $X$ syndrome. PLOS One. 2017;12(3):e0174301.

41. Klop B, Elte JW, Cabezas MC. Dyslipidemia in obesity: mechanisms and potential targets. Nutrients. 2013;5(4):1218-40.

42. Pal $\mathrm{S}$, et al. Identification of lipoproteins of intestinal origin in human atherosclerotic plaque. Clin Chem Lab Med. 2003;41(6):792-5. 